\title{
The metamorphosis of Supernova SN 2008D/XRF 080109: a link between Supernovae and GRBs/Hypernovae
}

\author{
Accepted by Science \\ Paolo A. Mazzali ${ }^{1,2,3,4 *}$, Stefano Valenti ${ }^{5,6}$, Massimo Della Valle ${ }^{7,8,9}$, \\ Guido Chincarini ${ }^{10,11}$, Daniel N. Sauer ${ }^{1}$, Stefano Benetti ${ }^{2}$, Elena Pian ${ }^{12}$, \\ Tsvi Piran ${ }^{13}$, Valerio D'Elia ${ }^{14}$, Nancy Elias-Rosa ${ }^{1}$, Raffaella Margutti ${ }^{10}$, \\ Francesco Pasotti ${ }^{10}$, L. Angelo Antonelli ${ }^{14}$, Filomena Bufano ${ }^{2}$, \\ Sergio Campana ${ }^{11}$, Enrico Cappellaro ${ }^{2}$, Stefano Covino ${ }^{11}$, \\ Paolo D'Avanzo ${ }^{11}$, Fabrizio Fiore ${ }^{14}$, Dino Fugazza ${ }^{11}$, Roberto Gilmozzi ${ }^{8}$, \\ Deborah Hunter $^{5}$, Kate Maguire ${ }^{5}$, Elisabetta Maiorano ${ }^{15}$, Paola Marziani ${ }^{2}$, \\ Nicola Masetti ${ }^{15}$, Felix Mirabel ${ }^{16}$, Hripsime Navasardyan ${ }^{2}$, \\ Ken'ichi Nomoto ${ }^{3,4,17}$, Eliana Palazzi ${ }^{15}$, Andrea Pastorello ${ }^{5}$, \\ Nino Panagia ${ }^{18,19}$, L.J. Pellizza ${ }^{20}$, Re'em Sari ${ }^{13}$, Stephen Smartt ${ }^{5}$, \\ Gianpiero Tagliaferri ${ }^{11}$, Masaomi Tanaka ${ }^{3}$, Stefan Taubenberger ${ }^{1}$, \\ Nozomu Tominaga ${ }^{3}$, Carrie Trundle ${ }^{5}$, Massimo Turatto ${ }^{19}$
}


${ }^{1}$ Max-Planck Institut für Astrophysik, Karl-Schwarzschild-Str.1, 85748 Garching, Germany

${ }^{2}$ Istituto Nazionale di Astrofisica-OAPd, vicolo dell'Osservatorio, 2, I-35122 Padova, Italy

${ }^{3}$ Department of Astronomy, School of Science, University of Tokyo, Bunkyo-ku, Tokyo 113-0033, Japan

${ }^{4}$ Research Center for the Early Universe, School of Science, University of Tokyo, Bunkyoku, Tokyo 113-0033, Japan

${ }^{5}$ Astrophysics Research Centre, School of Maths and Physics, Queen's University, Belfast, BT7 1NN, Northern Ireland, UK

${ }^{6}$ Dipartimento di Fisica, Universita' di Ferrara, via G. Saragat 1, 44100 Ferrara, Italy

${ }^{7}$ Istituto Nazionale di Astrofisica, Capodimonte Astronomical Observatory, Salita Moiariello 16, I-80131 Napoli, Italy

${ }^{8}$ European Southern Observatory, Karl-Schwarzschild-Str.2, D-85748 Garching, Germany

${ }^{9}$ International Center for Relativistic Astrophysics Network, Piazzale della Repubblica 10, I-65122 Pescara, Italy

${ }^{10}$ Department of Physics, Universita' di Milano-Bicocca, Piazza delle Scienze 3, I-20126 Milano, Italy

${ }^{11}$ Istituto Nazionale di Astrofisica, Brera Astronomical Observatory, Via E. Bianchi 46, I-23807 Merate (LC), Italy

${ }^{12}$ Istituto Nazionale di Astrofisica-OATs, Via Tiepolo 11, I-34131 Trieste, Italy

${ }^{13}$ The Racah Institute of Physics, Hebrew University, Jerusalem 91904, Israel

${ }^{14}$ Istituto Nazionale di Astrofisica, Rome Astronomical Observatory, Via di Frascati 33, I-00040 Monte Porzio Catone, Italy

${ }^{15}$ Istituto Nazionale di Astrofisica, Istituto di Astrofisica Spaziale e Fisica Cosmica, Via P. Gobetti 101, I-40129 Bologna, Italy

${ }^{16}$ European Southern Observatory, Alonso de Cordova 3107, Santiago, Chile

${ }^{17}$ Institute for the Physics and Mathematics of the Universe, University of Tokyo, Kashiwa, Chiba 277-8582, Japan

${ }^{18}$ Space Telescope Science Institute, Baltimore, MD, USA

${ }^{19}$ Istituto Nazionale di Astrofisica, Catania Astronomical Observatory, Via S. Sofia 78, I-95123 Catania, Italy

${ }^{20}$ Instituto de Astronomía y Física del Espacio, C.C. 67, Buenos Aires, Argentina

*To whom correspondence should be addressed; E-mail: mazzali@mpa-garching.mpg.de 
The only supernovae (SNe) to have shown early $\gamma$-ray or X-ray emission thus far are overenergetic, broad-lined Type Ic SNe (Hypernovae - HNe). Recently, SN 2008D shows several novel features: (i) weak XRF, (ii) an early, narrow optical peak, (iii) disappearance of the broad lines typical of SN Ic HNe, (iv) development of He lines as in SNe Ib. Detailed analysis shows that SN 2008D was not a normal SN: its explosion energy $\left(\mathrm{E} \approx 6 \cdot 10^{51} \mathrm{erg}\right)$ and ejected mass $\left(\sim 7 M_{\odot}\right)$ are intermediate between normal $\mathrm{SNe}$ Ibc and HNe. We derive that SN 2008D was originally a $\sim 30 M_{\odot}$ star. When it collapsed a black hole formed and a weak, mildly relativistic jet was produced, which caused the XRF. SN 2008D is probably among the weakest explosions that produce relativistic jets. Inner engine activity appears to be present whenever massive stars collapse to black holes.

On 2008 January 9.57 UT the X-Ray Telescope (XRT) on board Swift detected a weak X-ray Flash (XRF 080109) in the galaxy NGC2770(1). Optical follow-up revealed the presence of a supernova coincident with the XRF [SN 2008D; RA(2000) = 0909 30.625; Dec $(2000)=+3308$ 20.16](2). We detected SN 2008D photometrically from Asiago Observatory on 10.01 January 2008 UT, only 10.5 hours after the Swift detection. Early spectra showed broad absorption lines superposed on a blue continuum, and lacked hydrogen or helium lines(3). Accordingly, SN 2008D was classified as a broad-lined SN Ic(4). $\mathrm{SNe}$ of this type are sometimes associated with Gamma-ray Bursts (GRB, Refs. 5,6) or XRFs $(7,8)$. The spectra resembled those of the XRF-SN 2006aj (8) or the non-GRB HN SN 2002ap (9) (Fig. 3, top), but a comparison suggests that SN 2008D was highly reddened: we estimate that $E(B-V)_{t o t}=0.65 \mathrm{mag}$ (see SOM).

The host galaxy of XRF 080109/SN 2008D, NGC 2770 [redshift $z=0.006494$, distance $31 \mathrm{Mpc}]$, is a spiral galaxy similar to the Milky Way, M31, or ESO 184-G82, the host of SN 1998bw/GRB 980425. NGC 2770 has roughly solar metallicity and a moderate starformation rate, $\sim 0.5 M_{\odot} \mathrm{yr}^{-1}$ (see $\mathrm{SOM}$ ). In contrast, typical host galaxies of GRBs are small, compact, somewhat metal-poor, and highly star-forming(10).

In addition to the weak XRF, SN 2008D shows a number of peculiar features, most of which are new. The optical light curve had two peaks (Fig. 1): a first, dim maximum $(V \approx 18.4)$ was reached less than 2 days after the XRF. After a brief decline the luminosity increased again, reaching principal maximum $(V=17.37) \sim 19$ days after the XRF. An 18-20 day risetime is typical of GRB-HNe: normal SNe Ic reach maximum in 10-12 days. Few stripped-envelope SNe have very early data, and in GRB-HNe a first peak may be masked by the afterglow light. A first narrow optical peak was only seen in the Type Ib SN 1999ex (SNe Ib are similar to SNe Ic but show strong helium lines, Ref. 4), the Type IIb SN 1993J (SNe IIb are similar to SNe Ib but still have some hydrogen), and the Type Ic XRF-SN 2006aj. When it was discovered, SN 1999ex was dropping from a phase of high luminosity(11). It reached principal maximum $\sim 20$ days later, as did SN 2008D.

Another novel feature is the spectral metamorphosis (Fig. 2). Unlike SNe 2006aj and 2002ap, the broad absorptions did not persist. As they disappeared, He I lines developed (12). By principal maximum SN 2008D had a narrow-lined, Type Ib spectrum (Fig. 3, bottom). 
Broad lines require material moving with velocity $v>0.1 c$, where $c$ is the speed of light (13). Their disappearance implies that the mass moving at high velocities was small.

Late development of He I lines, previously seen only in SN 2005bf (14), is predicted by theory (15). Helium levels have high excitation potentials, exceeding the energy of thermal photons and electrons. Excitation can be provided by the fast particles produced as the $\gamma$-rays emitted in the decay chain of ${ }^{56} \mathrm{Ni}$ thermalize (16). This is the process that makes SNe shine. In the first few days after explosion thermalization is efficient because of the high densities and not enough particles are available to excite helium. Only when density drops sufficiently can more particles escape the ${ }^{56} \mathrm{Ni}$ zone and excite helium.

We reproduced the spectral evolution and the light curve of SN 2008D after the first narrow peak using a model with $M_{\mathrm{ej}} \sim 7 M_{\odot}$ and spherically symmetric $E \sim 6 \cdot 10^{51} \mathrm{erg}$, of which $\sim 0.03 M_{\odot}$, with energy $\sim 5 \cdot 10^{50} \mathrm{erg}$, are at $v>0.1 c$ (Figs. 1, 3, and SOM). Our light curve fits indicate that SN 2008D synthesised $\sim 0.09 M_{\odot}$ of ${ }^{56} \mathrm{Ni}$, like the non-GRB HN SN Ic 2002ap (9) and the normal SN Ic 1994I (17) but much less than the luminous GRB-HN SN 1998bw (6). The rapid rise in luminosity following the first peak requires that some ${ }^{56} \mathrm{Ni}\left(0.02 M_{\odot}\right)$ was mixed uniformly at all velocities $>9000 \mathrm{~km} \mathrm{~s}^{-1}$. This is a typical feature of $\mathrm{HNe}$, and indicates an aspherical explosion (18). Asphericity may affect our estimate of the energy, but not the ${ }^{56} \mathrm{Ni}$ mass (19).

Comparing the mass of the exploding He-star that we derived with evolutionary models of massive stars, we find that the progenitor had main sequence mass $\sim 30 M_{\odot}$. A star of this mass is likely to collapse to a black hole, as do GRB/SNe (20). So, SN 2008D shared several features of GRB/HNe. However, all SNe with GRBs or strong XRFs initially had velocities higher than SN 2008D or SN 2002ap (Fig. S3) and never showed helium. Had the He layer not been present in SN 2008D, the explosion energy would have accelerated the inner core to higher velocities, and broad lines may have survived.

The characterizing features of SN 2008D (weak XRF, first narrow optical peak, initially broad-lined SN Ic spectrum that later transformed into a narrow-lined SN Ib spectrum) may be common to all SNe Ib, or at least a significant fraction of them, and maybe some $\mathrm{SNe} I c$, which however contain little or no helium. The light curves of various SNe Ib are rather similar (21). The first peak was observed only for SN 1999ex, but lack of X-ray monitoring probably prevented the detection of more weak XRFs and the early discovery of the associated SNe. On the other hand SN 2008D, and possibly most SNeIb, was more energetic than normal core-collapse SNe, including most SNe Ic.

Type II SNe in late Spiral/Irr galaxies (the typical Hubble type of GRB hosts) are about 6 times more frequent than SNeIb (22). Although the serendipitous discovery of an SN Ib by XRT may be a statistical fluctuation, it may also suggest that the soft X-ray emission accompanying SN 2008D is typical of overenergetic SNe Ib, and absent (or very weak) in normal core-collapse SNe.

The X-ray spectrum of SN 2008D (in total 500 photons) can be fitted with either a simple power-law indicating a non-thermal emission mechanism or a combination of a hot black body $\left(T=3.8 \cdot 10^{6} \mathrm{~K}\right)$ and a power law. In the latter case, the unabsorbed luminosity of the black-body component is a small fraction of the total X-ray luminosity. The high temperature and low luminosity $\left(L=1.1 \cdot 10^{43} \mathrm{erg} \mathrm{s}^{-1}\right)$ of the black-body component at first peak ( $\sim 100 \mathrm{~s}$ after the onset of the XRF) imply an emitting radius $R_{\mathrm{ph}} \sim 10^{10} \mathrm{~cm}$ (see SOM, Section 4). This is at least one order of magnitude smaller than the size of 
Wolf-Rayet stars, the likely progenitors of SNe Ibc.

The X-ray flare and the first optical peak are most likely associated (23). The timescale of the first optical peak may suggest that it was related to shock breakout. A signature of shock breakout is a hot black-body X-ray spectrum immediately after the explosion. Thermal X-ray emission was suggested for SN 2006aj (24), while no X-ray data are available for SN 1999ex. The model of Ref. 23 uses a spherical configuration and a black-body component at $\sim 0.1 \mathrm{keV}$, below the XRT energy range. This yelds a large radius, which they explain invoking the presence of a dense surrounding medium that bulk-Comptonize the shock breakout emission to higher energies, producing the power-law spectrum observed by XRT between 0.3 and $10 \mathrm{keV}$.

On the other hand, the angular size of an emitting area with radius $R_{\mathrm{ph}} \sim 10^{10} \mathrm{~cm}$ is typical of GRB jets. This leads naturally to an alternative scenario, that we propose here: XRF 080109 was the breakout of a failed relativistic jet powered by a central engine as in GRBs. The jet failed because its energy was initially low or because it was damped by the He layer, which is absent in GRB-HNe, or both. The presence of a jet is supported by our conclusion that a black hole was probably formed when the star collapsed. The marginal breakout of the jet produced thermal X-rays and relativistic particles that caused the power-law X-ray component. It also caused the first optical peak: the timescale of the first peak and the X-ray flare and the corresponding radii and temperature are consistent with emission from rapidly expanding, adiabatically cooling material. The weakness of the jet resulted in the low X-ray flux and the small amount of material with $v>0.1 c$. The failed jet contributed anisotropically to the SN kinetic energy. Lateral spreading of the ejecta with $v>0.1 c$ leads to an angular size larger than the X-ray-emitting region, which is needed to produce the observed broad lines. The small amount of high-velocity material moving along our line-of-sight may also indicate that we viewed the explosion significantly off-axis. This can be tested by polarization or line profiles studies at late times, as in SN 2003jd (25). The jet will spread further after breakout and it could dominate the radio emission at later times.

The scenario we propose implies that GRB-like inner engine activity exists in all black hole-forming SNe Ibc (26). SN 2008D (and probably other SNe Ib) has significantly higher energy than normal core-collapse SNe, although less than GRB/HNe. Therefore, it is unlikely that all SNe Ibc, and even more so all core-collapse SNe produce a weak X-ray flash similar to XRF 080109. The presence of high-energy emission (GRB, XRF) depends on the jet energy and the stellar properties. Only massive, energetic, stripped SNe Ic (HNe) have shown GRBs. In borderline events like SN 2008D only a weak, mildly relativistic jet may emerge, because the collapsing mass is too small and a He layer damps the jet. For even less massive stars that still collapse to a black hole producing a less energetic explosion (e.g. SN 2002ap) no jet may emerge at all. Stars that only collapse to a neutron star are not expected to have jets. SN 2008D thus links events that are physically related but have different observational properties.

\section{References and Notes}

1. Berger, E., \& Soderberg, A.M., GCN 7159 (2008). 
2. Deng, J., \& Zhu, Y., GCN 7160 (2008).

3. Valenti, S., et al., GCN 7171 (2008).

4. Filippenko, A.V., Ann. Rev. Astron. Astrophys. 35, 309 (1997).

5. Galama, T. J., et al., Nature 395, 670 (1998).

6. Iwamoto, K., et al., Nature 395, 672 (1998).

7. Pian, E., et al., Nature 442, 1011 (2006).

8. Mazzali, P. A., et al., Nature 442, 1018 (2006).

9. Mazzali, P. A., et al., Astrophys. J. 572, L61 (2002).

10. Fruchter, A.S., et al., Nature 441, 463 (2006).

11. Stritzinger, M., et al., Astron. J. 124, 2100 (2002).

12. Modjaz, M., Chornock, R., Foley, R.J., Filippenko, A.V., Li W., \& Stringfellow G., GCN 7212 (2008).

13. Mazzali, P. A., Iwamoto, K., Nomoto, K., Astrophys. J. 545, 407 (2002).

14. Tominaga, N., et al., Astrophys. J. 633, L97 (2005).

15. Mazzali, P. A., \& Lucy, L.B., Mon. Not. R. Astron. Soc. 295, 428 (1998).

16. Lucy, L.B., Astrophys. J. 383, 308 (1991).

17. Sauer, D., et al., Mon. Not. R. Astron. Soc. 369, 1939 (2006).

18. Maeda, K., et al., Astrophys. J. 593, 931 (2003).

19. Maeda, K., Mazzali, P.A.,, \& Nomoto, K., Astrophys. J. 645, 1331 (2006).

20. MacFadyen, A. E., \& Woosley, S. E., Astrophys. J. 524, 262 (1999).

21. Clocchiatti, A., \& Wheeler, J.C., Astrophys. J. 491, 375 (1997).

22. Cappellaro, E., Evans, R., Turatto, M., A $\mho A$ 351, 459 (1999).

23. Soderberg, A.M., et al., Nature 453, 469 (2008).

24. Campana, S., et al., Nature 442, 1008 (2006).

25. Mazzali, P.A., et al., Science 308, 1284 (2005).

26. Maeda, K., et al., Science 319, 1220 (2008)

27. Arnett, W. D., Astrophys. J. 253, 785 (1982).

28. Mazzali, P.A., et al., Astrophys. J. 645, 1323 (2006).

29. Cappellaro, E., et al., Astron. Astrophys. 328, 203 (1997). 


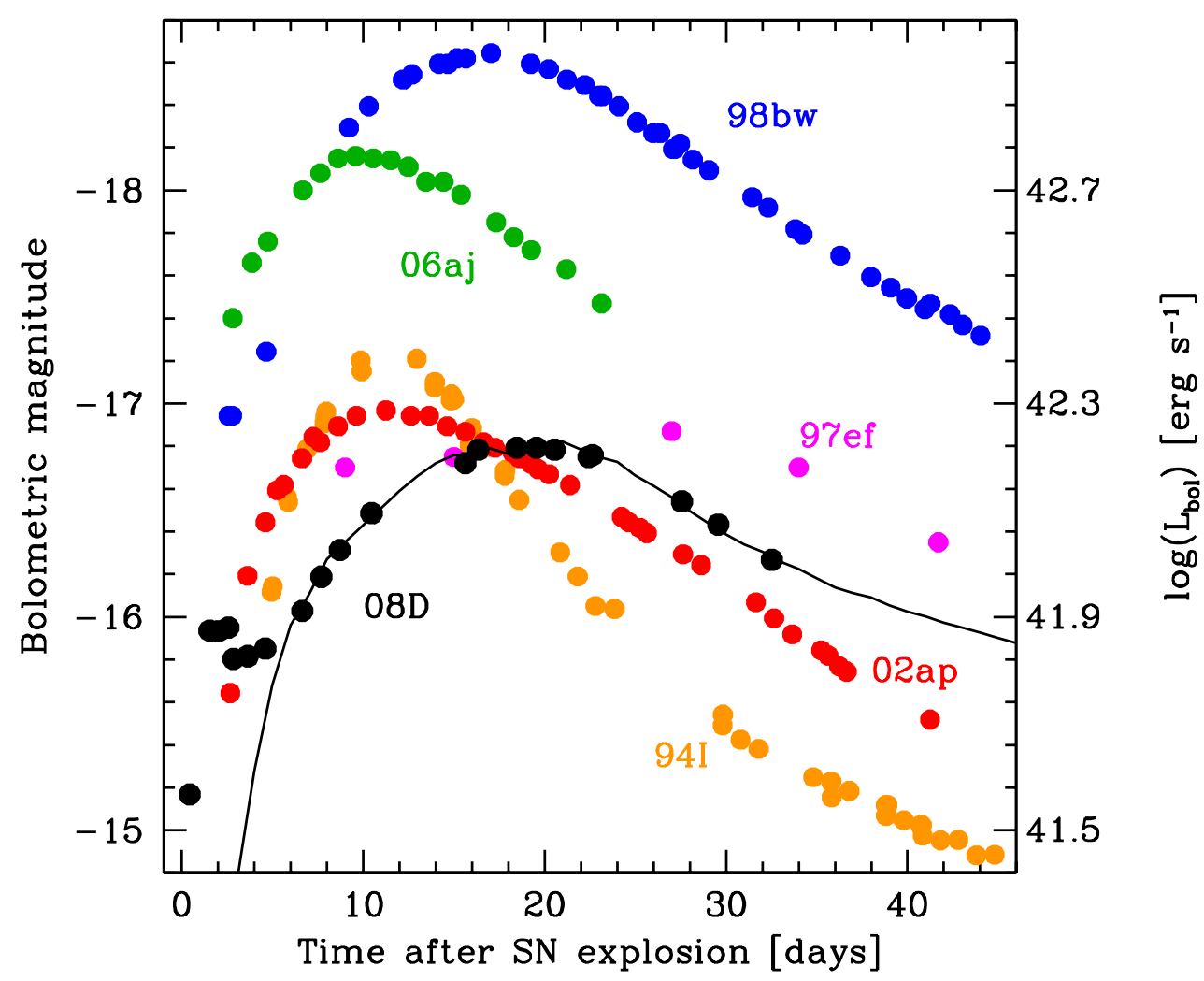

Figure 1: The light curves of SN 2008D and of other Type Ibc SNe. The shape of the light curve of SN 2008D is similar to that of SN 1998bw and other GRB/HNe, and comparable to the non-GRB HN SN 1997ef, but much broader than the XRF/SN 2006aj or the normal SN Ic 1994I. This similarity suggests a comparable value of the quantity $M_{\mathrm{ej}}{ }^{3} / E$, where $M_{\mathrm{ej}}$ is the mass ejected and $E$ the explosion kinetic energy(27). All known SNe Ic with a broad light curve ejected a large mass of material(28). Large values of $M_{\mathrm{ej}}$ and $E$ are also suggested by the presence of He moving at $v \sim 10000 \mathrm{~km} \mathrm{~s}^{-1}$ : the velocity of He in SN 2005bf was lower(14). The light curve of SN 1999ex, which is similar to that of SN 2008D, was fitted reasonably well by a He-star explosion model with $M_{\mathrm{ej}} \sim 5 M_{\odot}, E \sim 3 \cdot 10^{51} \mathrm{erg}(11)$. Such a model would also match the light curve of SN 2008D, but it probably would not reproduce the broad lines that characterize the early spectra. This would require a model containing some high-velocity material, leading to a larger $E$ without significantly affecting the value of $M_{\mathrm{ej}}$ or the light curve shape. The line shows a synthetic bolometric light curve computed with a Montecarlo code(29) for a model with $M_{\mathrm{ej}} \sim 7 M_{\odot}$, $E \sim 6 \cdot 10^{51} \mathrm{erg}$. The model does not address the physics that may be responsible for the first narrow light curve peak, but only the main peak, which is due to diffusion of radiation in the SN envelope following the deposition of $\gamma$-rays and positrons emitted in the decay chain ${ }^{56} \mathrm{Ni}$ to ${ }^{56} \mathrm{Co}$ and ${ }^{56} \mathrm{Fe}$. 


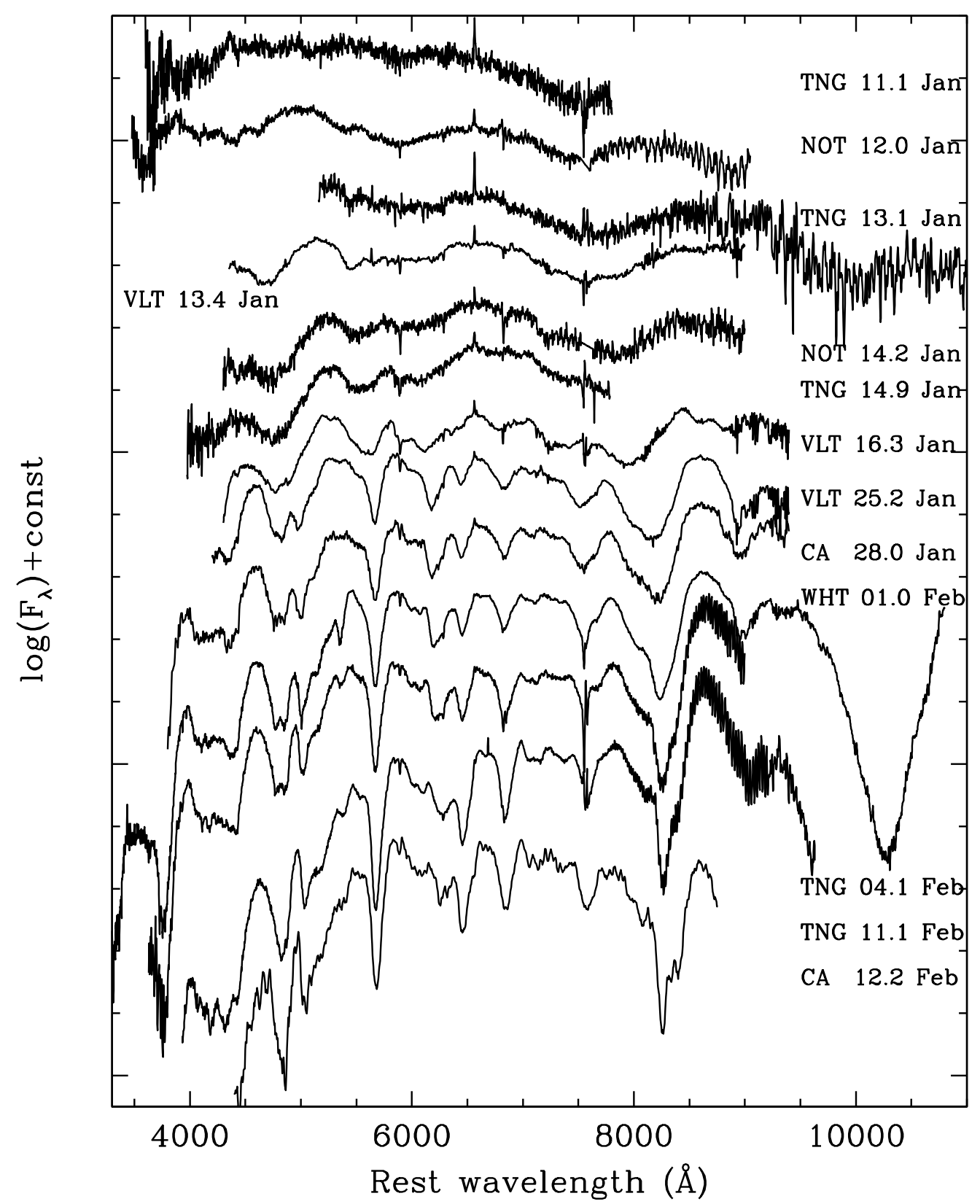

Figure 2: Spectral evolution of SN 2008D. In the early phase the strongest features are broad Fe complexes in the blue $(\sim 4000-5000 \AA)$, the Si II-dominated feature near $6000 \AA$, and Ca II lines, both in the near UV (H\&K) and in the near IR (the IR triplet near $8500 \AA)$. On the other hand O I $7774 \AA$, which is strong in all HNe as well as in all SNe Ibc, is conspicuously missing. Starting 15 Jan, lines begin to become narrow. In the later spectra, taken near maximum, He I lines have developed. The strongest isolated lines are $\lambda 6678 \AA$, seen near $6500 \AA$, and $\lambda 7065 \AA$, seen near $6900 \AA$. Both lines indicate a helium velocity of $\sim 10000 \mathrm{~km} \mathrm{~s}^{-1}$. The other strong optical lines of He I are blended: $\lambda 5876 \AA$ is blended with Na I D, near $5600 \AA$, and $\lambda 4471 \AA$ is blended with the broad Fe II trough near $4200 \AA$. 


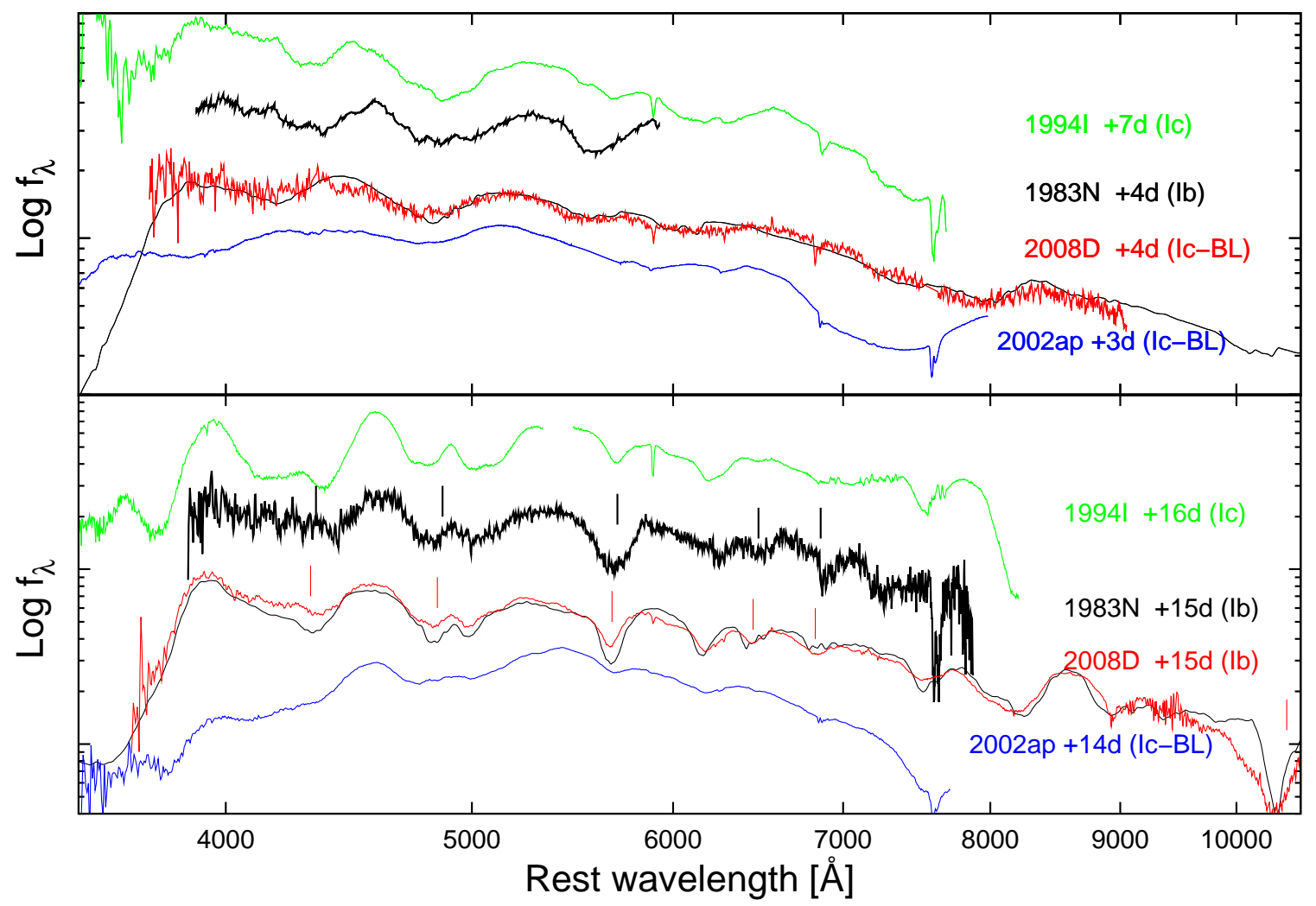

Figure 3: The spectra of SN 2008D compared to those of other Type Ibc SNe and to simulations. Near the first peak (top), SN 2008D has a broad-lined spectrum similar to that of SN 2002ap, a broad-lined SN without a GRB(9), but different from both the normal SN Ic 1994I and the SN Ib 1983N. At the time of the main light curve peak (bottom), the spectrum of SN 2008D has narrow lines like SNe 1994I and 1983N, while SN 2002ap and other HNe retain broad features throughout their evolution. Also, SN 2008D developed He lines (vertical ticks). At this epoch, the spectra of SNe 2008D, 1983N, and 1999ex are similar. Synthetic spectra are overlaid on the two SN 2008D spectra (see SOM). 


\section{Supplementary Information}

\section{Optical and infrared observations}

SN 2008D was observed photometrically and spectroscopically with a number of telescopes.

Low-resolution spectra of SN 2008D have been obtained with the European Southern Observatory (ESO) 8.2m Very Large Telescope (VLT)-UT2, equipped with FORS2; the $4.2 \mathrm{~m}$ William Herschel Telescope (WHT) equipped with ISIS; the $3.6 \mathrm{~m}$ Telescopio Nazionale Galileo (TNG), equipped with DOLORES for optical observations and with NICS for near-IR observations; the 3.6m ESO-New Technology Telescope (NTT), equipped with EMMI; the 2.5m Nordic Optical Telescope (NOT), equipped with ALFOSC; the 2.2m Calar Alto telescope (CA), equipped with CAFOS; the $2.0 \mathrm{~m}$ Liverpool Telescope (LT), equipped with RATCam; the 1.82m Asiago-Ekar telescope (As1.82m), equipped with AFOSC; the 0.60m Rapid Eye Mount telescope (REM), equipped with ROSS for optical observations and with REMIR for near-IR observations.

Tables S1 and S2 report the log of the observations covering the first month after the $\mathrm{XRF}$, with details on the acquisition, and the photometric results. Column 1 gives the observation date, Column 2 the telescope and instrument used, Column 3 the observing setup, Column 4 the average seeing during the photometric acquisition (Table S1) and the spectral resolution (Table S2), Column 5 the observed V magnitude (Table S1) and the spectrophotometric standard used to calibrate the spectra (Table S2). The photometric errors are $1 \sigma$ uncertainties.

Monochromatic light curves are shown in Fig. S1, and the spectral evolution in Fig. 2 in the main paper). 
Supplementary Table S1: Photometric observations of SN 2008D

\begin{tabular}{ccccc}
\hline \hline $\begin{array}{c}\text { Date } \\
\text { (2008 UT) }\end{array}$ & $\begin{array}{c}\text { Telescope+ } \\
\text { Instrument }\end{array}$ & Setup & $\begin{array}{c}\text { Seeing } \\
(\operatorname{arcsec})\end{array}$ & $\mathrm{V}$ \\
\hline 7.005 Jan & As1.82m+AFOSC & UBVRI & 2.0 & $<19.0$ \\
10.012 Jan & As1.82m+AFOSC & UBVRI & 2.5 & $19.10 \pm 0.06$ \\
11.213 Jan & TNG+NICS & JHK & 0.9 & - \\
12.168 Jan & NOT+ALFOSC & UBVRI & 2.0 & $18.41 \pm 0.10$ \\
13.006 Jan & TNG+NICS & JHK & 0.8 & - \\
13.212 Jan & TNG+DOLORES & UBVRI & 0.9 & $18.49 \pm 0.05$ \\
14.174 Jan & NOT+ALFOSC & UBVRI & 1.6 & $18.29 \pm 0.05$ \\
16.205 Jan & LT+RATCam & UBVRI & 1.9 & $18.03 \pm 0.04$ \\
17.270 Jan & LT+RATCam & UBVRI & 1.6 & $17.96 \pm 0.02$ \\
18.267 Jan & LT+RATCam & UBVRI & 1.9 & $17.79 \pm 0.04$ \\
20.024 Jan & LT+RATCam & UBVRI & 2.2 & $17.64 \pm 0.06$ \\
20.153 Jan & REM+REMIR & H & 3.5 & - \\
21.310 Jan & REM+REMIR & H & 2.9 & - \\
22.309 Jan & REM+REMIR & H & 2.9 & - \\
23.123 Jan & REM+REMIR & JK & 1.4 & - \\
25.197 Jan & VLT+FORS2 & BVRI & 1.0 & $17.36 \pm 0.04$ \\
25.906 Jan & LT+RATCam & UBVRI & 1.9 & $17.36 \pm 0.03$ \\
28.021 Jan & CA+CAFOSC & UBVRI & 1.2 & $17.33 \pm 0.05$ \\
28.987 Jan & TNG+NICS & JHK & 1.4 & - \\
29.128 Jan & LT+RATCam & UBVRI & 1.2 & $17.34 \pm 0.03$ \\
30.127 Jan & LT+RATCam & UBVRI & 2.9 & $17.35 \pm 0.04$ \\
31.239 Jan & REM+ROS+REMIR & RJHK & 3.0 & - \\
31.947 Jan & TNG+NICS & JHK & 3.1 & - \\
31.964 Jan & LT+RATCam & UBVRI & 1.3 & $17.38 \pm 0.05$ \\
\hline & & & & \\
\hline
\end{tabular}

Supplementary Table S1: Photometric observations of SN 2008D, continued

\begin{tabular}{ccccc}
\hline \hline $\begin{array}{c}\text { Date } \\
(2008 \mathrm{UT})\end{array}$ & $\begin{array}{c}\text { Telescope+ } \\
\text { Instrument }\end{array}$ & Setup & $\begin{array}{c}\text { Seeing } \\
(\operatorname{arcsec})\end{array}$ & $\mathrm{V}$ \\
\hline $01.160 \mathrm{Feb}$ & LT+RATCam & UBVRI & 1.3 & $17.43 \pm 0.03$ \\
05.111 Feb & REM+ROS+REMIR & RJHK & 3.2 & - \\
$06.114 \mathrm{Feb}$ & LT+RATCam & BVRI & 1.3 & $17.58 \pm 0.04$ \\
$08.162 \mathrm{Feb}$ & LT+RATCam & UBVRI & 2.1 & $17.74 \pm 0.03$ \\
$11.091 \mathrm{Feb}$ & TNG+DOLORES & UBVRI & 1.8 & $18.05 \pm 0.04$ \\
$13.091 \mathrm{Feb}$ & REM+ROS+REMIR & JK & 3.2 & - \\
\hline
\end{tabular}


Supplementary Table S2: Spectroscopic observations of SN 2008D

\begin{tabular}{lcccc}
\hline \hline $\begin{array}{c}\text { Date } \\
\text { 2008 UT })\end{array}$ & $\begin{array}{c}\text { Telescope+ } \\
\text { Instrument }\end{array}$ & Setup & $\begin{array}{c}\text { Resolution } \\
(\AA)\end{array}$ & standard \\
\hline 11.097 Jan & TNG+DOLORES & LR-B & 12 & HD93521 \\
12.045 Jan & NOT+ALFOSC & gm4 & 14 & Feige34 \\
13.056 Jan & TNG+NICS & IJ & 7 & Hip10559 \\
13.187 Jan & TNG+DOLORES & LR-R & 13 & Feige34 \\
13.392 Jan & VLT+FORS2 & 300V; 300I & 10 & HILT600 \\
14.194 Jan & NOT+ALFOSC & gm4 & 14 & G191-B2B \\
14.944 Jan & TNG+DOLORES & LR-B & 13 & Feige34 \\
16.280 Jan & VLT+FORS2 & 300V; 300I & 10 & LTT3864 \\
25.212 Jan & VLT+FORS2 & 300V-300I & 10 & HILT600 \\
28.033 Jan & CA+CAFOSC & b200 & 13 & Feige34 \\
28.270 Jan & NTT+EMMI & gm2 & 12 & Feige34 \\
28.935 Jan & TNG+NICS & IJ+HK & 7 & Hip10559 \\
28.966 Jan & As1.82m+AFOSC & gm4+gm2 & 25 & Feige34 \\
29.958 Jan & As1.82m+AFOSC & gm4+gm2 & 25 & Feige34 \\
31.957 Jan & TNG+NICS & IJ+HK & 7 & Hip10559 \\
01.002 Jan & WHT+ISIS & R300B+R158R & 10 & HD93521 \\
04.107 Feb & TNG+DOLORES & LR-B+LR-R & 12 & Feige34 \\
11.100 Feb & TNG+DOLORES & LR-B+LR-R & 11 & G191-B2B \\
12.185 Feb & CA+CAFOSC & b200 & 13 & Feige34 \\
\hline
\end{tabular}




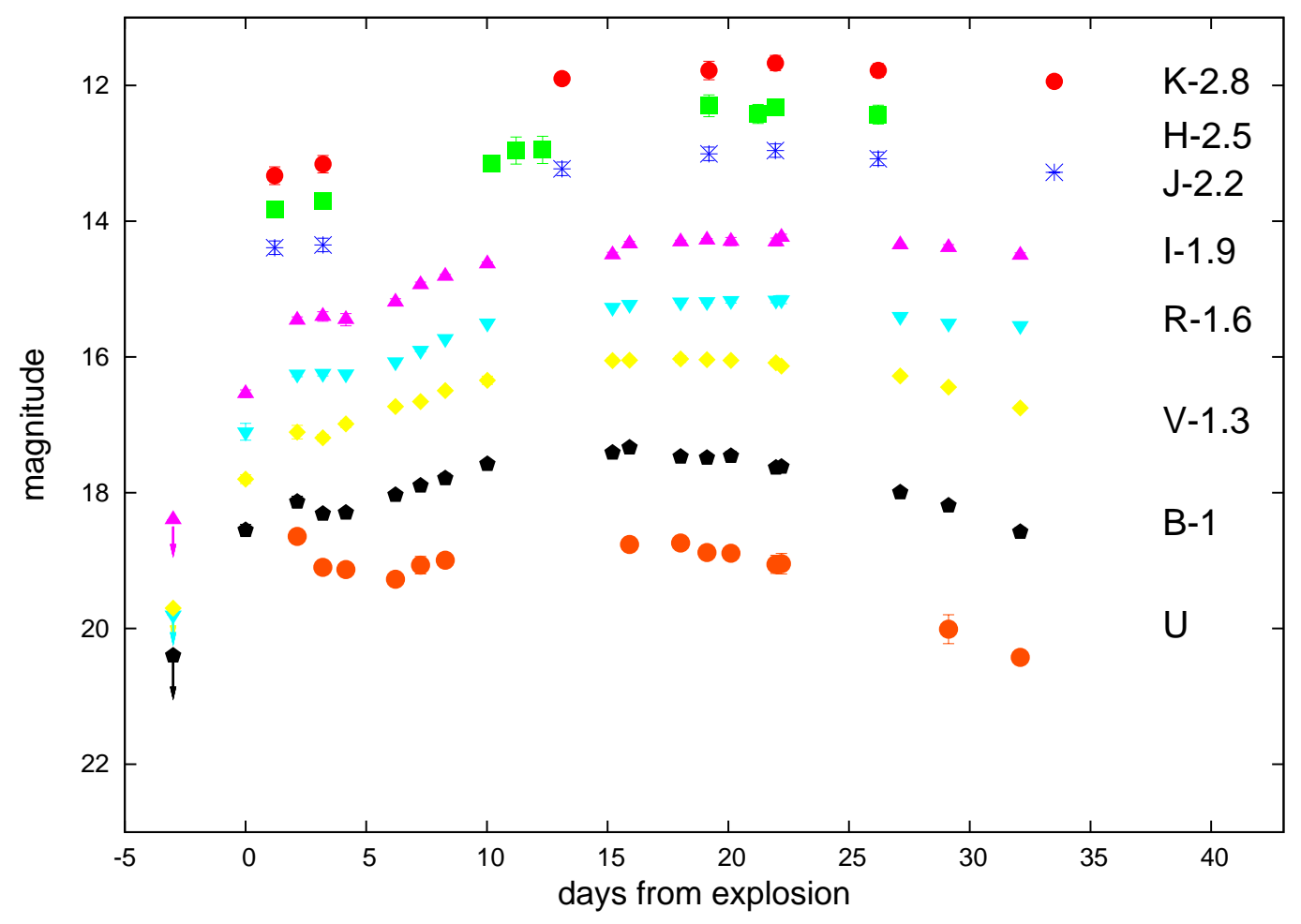

Figure 4: The monochromatic light curves of SN 2008D. 


\section{The properties of NGC 2770, the host of SN 2008D/XRF080109.}

NGC 2770 is an Sc galaxy of luminosity class III-IV, similar to the Milky Way (S1), and very different from the hosts of long $\mathrm{GRBs}(\mathrm{S} 2)$. Its nucleus is rather diffuse, and according to FIRST radio maps it does not coincide with any radio peak. The SDSS spectrum of the nucleus is typical of star-forming nuclei of late-type spiral galaxies: relatively narrow lines (FWHM $<200 \mathrm{~km} \mathrm{~s}^{-1}$ ) and diagnostic emission line ratios are consistent with photoionization by hot stars. A ratio $[\mathrm{O}$ III $] \lambda 5007 / \mathrm{H} \beta=0.68$, uncorrected because of the clearly visible $\mathrm{H} \beta$ absorption, makes NGC 2770 a galaxy with an $\mathrm{H}$ II nuclear spectrum(S3). Since the Balmer lines are mainly due to photoionization by hot stars, the star formation rate can be estimated from indicators such as the $\mathrm{H} \alpha$ flux $\left(0.2 M_{\odot} \mathrm{yr}^{-1}\right)$, IRAS fluxes $\left(0.45 M_{\odot} \mathrm{yr}^{-1}\right)$, or the radio luminosity at $1.4 \mathrm{GHz}\left(0.7 M_{\odot} \mathrm{yr}^{-1}\right)$. From the FIR Luminosity we derive(S4) a SN rate $\sim 0.01 \mathrm{SNe} \mathrm{yr}^{-1}$. These values indicate that NGC 2770 does not have a very high star formation luminosity. There is no strong evidence of a hidden non-thermal source, even at radio frequencies, as already shown by ref. S5, who failed to find a high surface brightness nuclear radio source in NGC 2770. 


\section{Estimate of the reddening to $\mathrm{SN} 2008 \mathrm{D}$ and derivation of the bolometric light curve.}

We estimate dust extinction towards SN 2008D from spectral comparison with other SNe Ibc. Using $E(B-V)=0.16$ for SN 1983N (S6), an extinction $E(B-V)_{\text {tot }}=0.65 \mathrm{mag}$ for SN 2008D makes the two SNe almost identical (Fig. 3 in the main paper). This value is confirmed by two independent checks: 1) it is compatible with the neutral hydrogen column density $6 \cdot 10^{21} \mathrm{~cm}^{-2}$ estimated from X-ray spectral fits assuming a Galactic gas-todust ratio $(7) ; 2$ ) it yields optical and infrared colour light curves similar to those of other $\mathrm{SNe} \mathrm{Ib} / \mathrm{c}$. Considering the uncertainties, we adopt for SN 2008D $E(B-V)_{t o t}=0.65 \pm 0.15$. Most of the extinction occurs in the host galaxy, since Galactic extinction is very weak $\left[E(B-V)_{\text {Gal }}=0.02\right.$ mag; ref. 8].

We computed the bolometric light curve using the data in Table 1 together with the measurements reported in refs. 9 and 10. 


\section{X-ray data and models}

The XRT light curve (Figure S2, top panel) was fitted with a function CountRate $=$ $N\left(t-t_{0}\right) \sim e^{-\alpha\left(t-t_{0}\right)}$, where $N=14.5 \pm 2.2$ and $\alpha=1.09 \pm 0.13$, yielding $\chi^{2} /$ d.o.f. $=$ $13.1 / 16$. From this fit, the onset of the XRF can be estimated to have occurred on $J D=2454475.06413$. The $\mathrm{X}$-ray flux reaches maximum in $\approx 92 \mathrm{~s}$, with an uncertainty of $\sim 30 \mathrm{~s}$. The decaying part of the X-ray curve can also be fitted with a power-law with index $\alpha=1.52 \pm 0.16$. The number of photons is too small to perform timeresolved spectroscopy. Instead, we show the time evolution of the hardness ratio $H R=$ $C R(1.5-10 \mathrm{keV}) / C R(0.3-1.5 \mathrm{keV}$ (Fig. S2, bottom panel). The spectrum hardens during the rise to maximum and softens afterwards, a typical behaviour of X-ray flares in GRBs and in FRED profiles.

The source is affected by pile-up only within a 3-pixel radius. Therefore we extracted the photons from an annular region with a 3-pixel inner radius and a 30-pixel outer radius, yielding a total of 539 photons.

The spectrum can be fitted with a simple power-law typical of GRBs, with photon in$\operatorname{dex} \Gamma=2.29_{-0.24}^{+0.26}$ and a host galaxy hydrogen column density of $N_{H}=6.42_{-1.18}^{+1.42} \cdot 10^{21} \mathrm{~cm}^{-2}$ $\left(\chi^{2} /\right.$ d.o.f. $\left.=21.1 / 23\right)$. Alternatively, a model with a black body with temperature $k T=0.20_{-0.20}^{+0.30} \mathrm{keV}$ superimposed on a power-law with photon index $\Gamma=2.21_{-0.93}^{+0.48}$, and a host galaxy hydrogen column density $N_{H}=6.96_{-2.71}^{+7.03} \cdot 10^{21} \mathrm{~cm}^{-2}$ yields $\chi^{2} /$ d.o.f. $=20.6 / 21$. The total intensity is $1.85 \times 10^{-10} \mathrm{erg} \mathrm{cm}^{-2} \mathrm{~s}^{-1}$, and the black body intensity is no more than $14 \%$ of it, thus the presence of a black body remains uncertain (11).

The X-ray luminosity can be computed using the conversion from count rate to flux 1 count $\mathrm{s}^{-1}=1.17 \cdot 10^{-10} \mathrm{erg} \mathrm{cm}^{-2} \mathrm{~s}^{-1}$, derived from the spectral power-law fit corrected for $N_{H}$ absorption. Over the $605 \mathrm{sec}$ of the X-ray event, after applying a PSF correction factor of 1.88, this yields a fluence of $1.1 \cdot 10^{-7} \mathrm{erg} \mathrm{cm}^{-2}$, corresponding to a total energy $1.3 \cdot 10^{46} \mathrm{erg}$. The peak luminosity is $\sim 8 \cdot 10^{43} \mathrm{erg} \mathrm{s}^{-1}$. Assuming that the black-body component is a constant fraction $(14 \%)$ of the total luminosity at any time, its luminosity, combined with the high black-body temperature $\left(3.8 \cdot 10^{6} \mathrm{~K}\right)$, implies an emitting radius $R_{\mathrm{ph}} \sim 10^{10} \mathrm{~cm}$, an order of magnitude smaller than the size of Wolf-Rayet stars. 

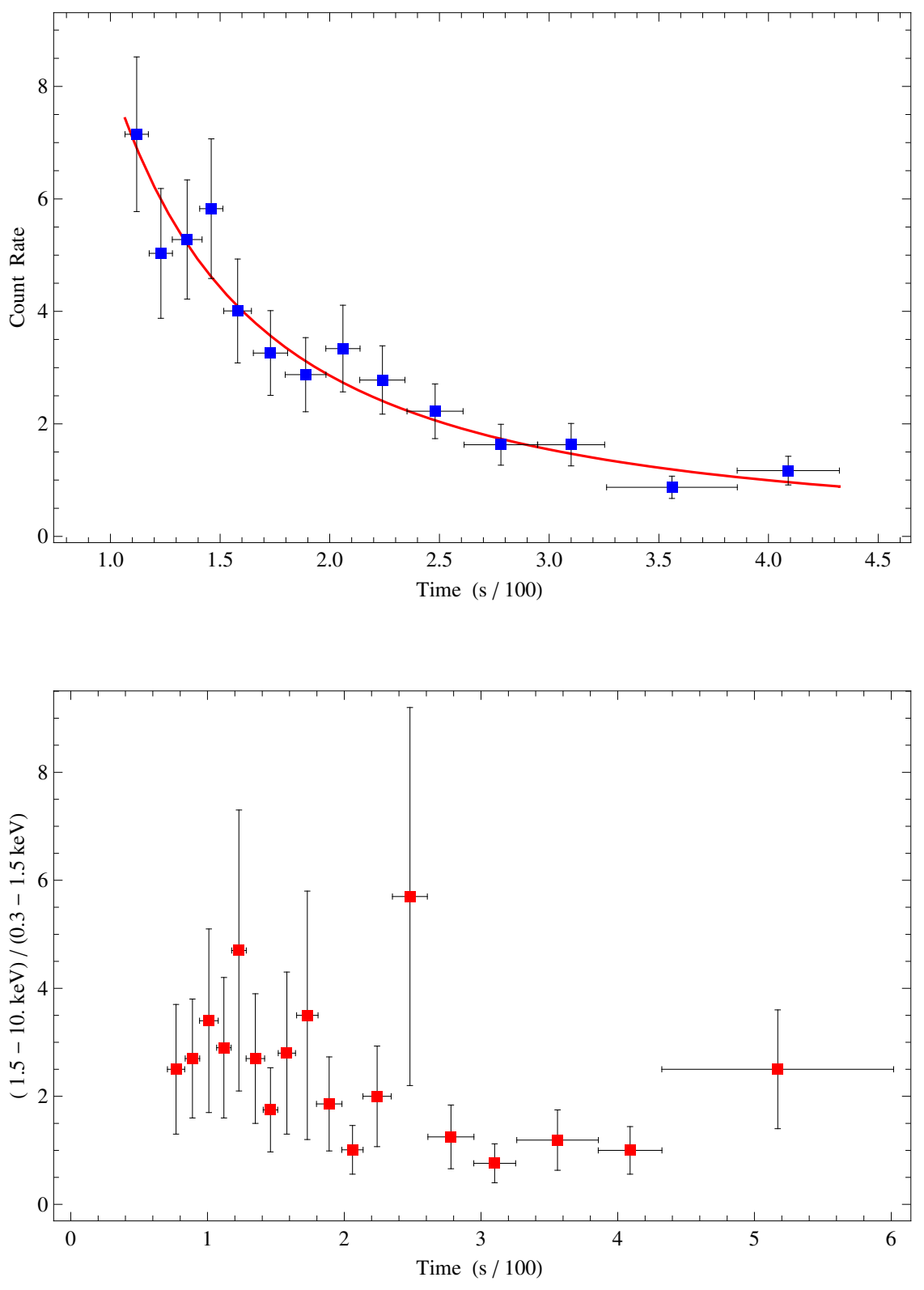

Figure 5: Top: the decaying part of the X-ray curve and a fit with a power-law with index $\alpha=1.52 \pm 0.16$; Bottom: time evolution of the hardness ratio $H R=C R(1.5-$ $10 \mathrm{keV}) / C R(0.3-1.5 \mathrm{keV})$. 


\section{Spectral modelling.}

To estimate the physical parameters that describe the supernova explosion we construct a series of radiative transfer models to derive synthetic spectra. As time elapses after the explosion of the supernova the ejecta expand and become diluted, progressively exposing deeper layers. Modelling the spectra as they evolve enables us to infer the structure of the ejected material. Among the parameters that can be determined in this way are the luminosity, the postion of the momentary photosphere, and the composition and velocity of the line-forming layers of the ejecta at each epoch.

For the spectral models we use a Monte Carlo radiative transfer code (S12, S13, S14). The code employs an approximate description of non-LTE suitable for the analysis of supernova spectra during the photospheric epochs while retaining physically meaningful relationships between model parameters. Here we use a version of the code that uses a depth-dependent composition structure (S15). For all models in the series a density structure $\rho(v)$ is adopted (for SNe a Hubble-like expansion law $r=v \cdot t$ allows us to use the ejecta velocity as a time-independent radial variable). Input parameters for each individual model are the luminosity at the given epoch, a lower boundary velocity, and the composition above this velocity. This lower boundary represents the pseudo-photosphere from which all radiation is assumed to emerge, which is a good approximation at early times. Lacking self-consistent explosion models we use a parameterized density structure which we constrain iteratively with the help of light curve and spectral models. Our model does not take into account non-thermal excitation and ionization by fast particles from the radioactive decay of ${ }^{56} \mathrm{Ni}$. This is a fair approximation for most elements but it fails for helium. Because of the high ionization potential of He I, non-thermal processes are the main contribution to the excitation of this elements. Therefore, we cannot selfconsistently derive the opacity of He I lines, which would anyway require a detailed model of the distribution of ${ }^{56} \mathrm{Ni}$ and of the geometry of the ejecta.

The very broad line features at early epochs, up to day 5 after explosion, indicate the presence of appreciable amounts of matter at high velocities above $v \sim 30,000 \mathrm{~km} \mathrm{~s}^{-1}$. Starting approximately 2008 January $15,(t=6 \mathrm{~d})$ the lines become narrower and the blueshifted high-velocity components disappears. This suggests a significant steepening of the density gradient at lower velocities. The last spectrum considered here was obtained on 2008 February $11(t=33 \mathrm{~d})$. For this model we use a lower boundary velocity of $7500 \mathrm{~km} \mathrm{~s}^{-1}$. The line widths of this last spectrum suggest that the density gradient flattens below a velocity of about $9000 \mathrm{~km} \mathrm{~s}^{-1}$. Deeper layers are not yet accessible to observations. Fig. S3 shows the time evolution of the photospheric velocity.

A rough estimate of the parameters is obtained from the similarity of SN 2008D to SNe 2002ap and 2006aj at the earliest times and to SN 1994I at later phases. The models of these objects (S16, S17, and S18) are used as a guideline. The evolution of synthetic spectra is shown in Fig. S4.

For the models shown here we use a broken power-law with a power-law index of $n=$ -2.0 in the region inside of $9000 \mathrm{~km} \mathrm{~s}^{-1}, n=-7.5$ between $9000 \mathrm{~km} \mathrm{~s}^{-1}$ and $17,000 \mathrm{~km} \mathrm{~s}^{-1}$, and $n=-5.5$ above $17,000 \mathrm{~km} \mathrm{~s}^{-1}$. We set the absolute density to match the observed spectral features, and obtain a total ejected mass of $\sim 7 M_{\odot}$ with a kinetic energy of $\sim 6 \times 10^{51} \mathrm{erg}$, of which a mass $\sim 0.03 M_{\odot}$ with a $E \sim 5 \times 10^{50} \mathrm{erg}$ is located at $v>$ 
$30,000 \mathrm{~km} \mathrm{~s}^{-1}$.

The first spectrum we consider here was taken on 12 January, 3 days after the explosion. An interesting feature of the spectra is the absence of a strong $\mathrm{O}$ I absorption at $\sim 7300 \AA$. The $\mathrm{O}$ I lines at $7774 \AA$ that are normally responsible for this feature are very strong. Therefore we can only accommodate small amounts of oxygen in the outer layers of the ejecta. At early epochs oxygen could be so highly ionized that O I lines disappear, but the presence of CaII and Fe II in the spectra makes this hypothesis unlikely because those species should not be present in an environment where oxygen is mostly ionized.

Overall, we can model the spectra with only a mild variation of the composition with depth. Spectra before maximum exhibit broad but shallow features of Ca, Si, and Fegroup elements suggesting the presence of only small amounts of absorbing material. In the outer part of the ejecta above $v \sim 14,000 \mathrm{~km} \mathrm{~s}^{-1}$ we assume a composition dominated by He. The attenuation of the flux in the blue requires the presence of some Ti and other Fe-group elements that do not produce strong individual line features but block radiation in the blue and UV through a large number of overlapping weaker lines.

At epochs around and after maximum the spectra show distinctly narrower line features that can be attributed the HeI, O I, CaII and Fe II. There is also some indication for $\mathrm{C}_{\mathrm{I}}$ in the red and infrared region (S19). In the inner part we assume a composition where $\mathrm{C}$ and $\mathrm{O}$ dominate with a slightly enhanced contribution from heavy elements, likely including some decay products of radioactive ${ }^{56} \mathrm{Ni}$. 


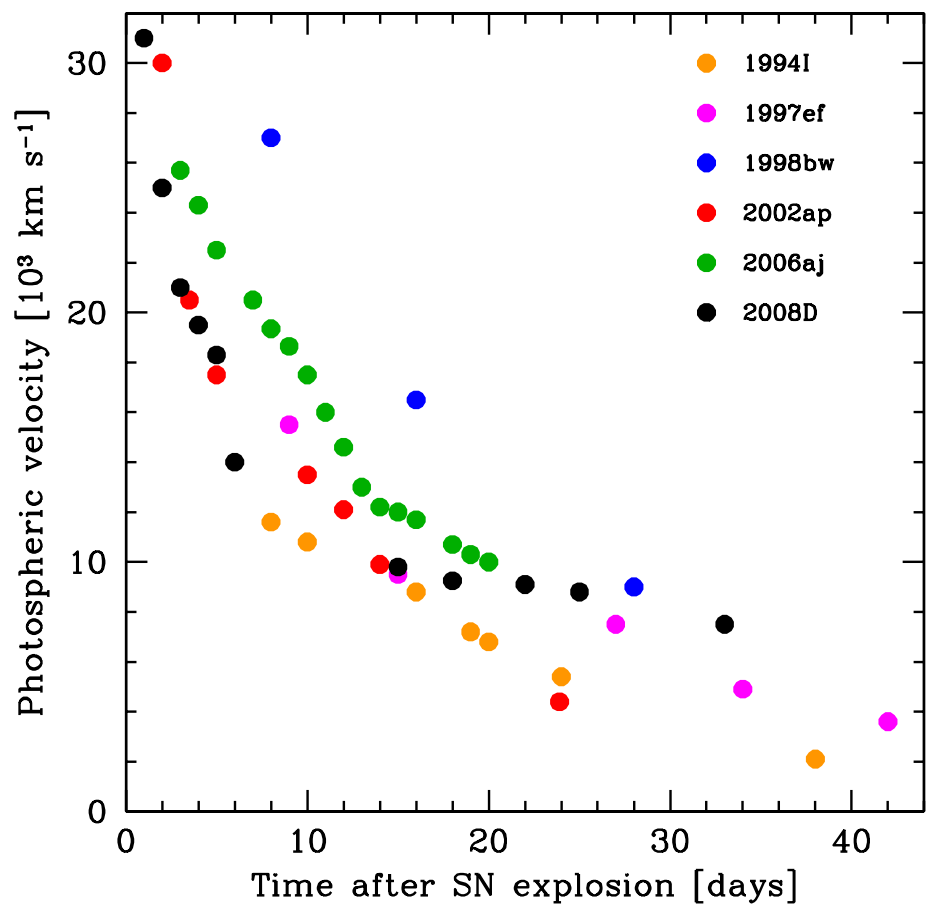

Figure 6: The temporal evolution of the lower boundary (photosphere) velocity used in the models for SN 2008D compared to the cases of other SNe Ic. SN 2008D starts out with a very high velocity, like SN 2002ap, then transitions to lower velocities like SN 1994I. This is the phase when broad lines disappear. At later times the evolution is slower than that of SN 1994I, indicating a large mass with a small density gradient in the inner layers, similar to SN 1997ef. Only SNe with velocities larger than SNe2008D or 2002ap were accompanied by a GRB or an XRF. 


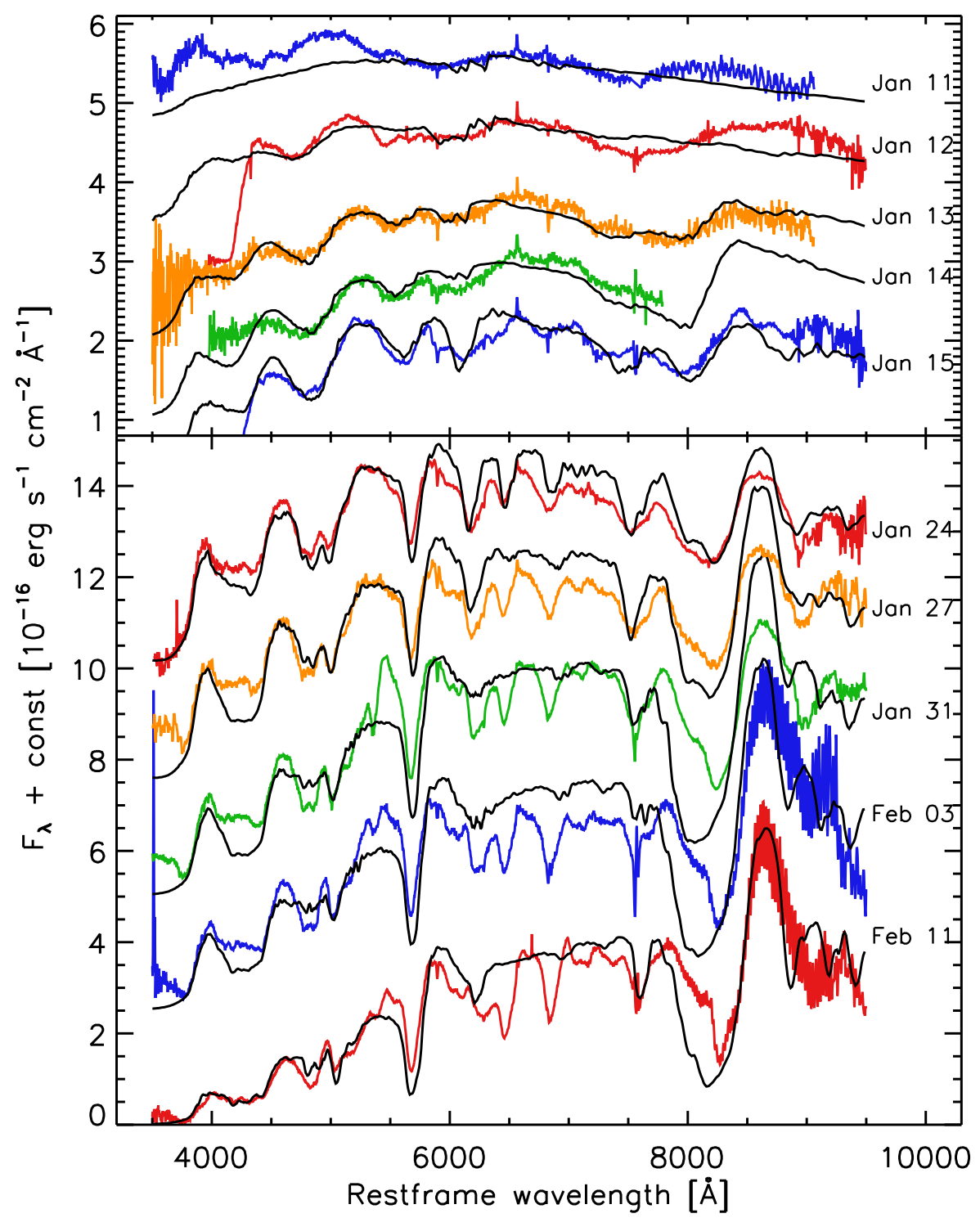

Figure 7: Series of model spectra for the sequence of early time spectra of SN 2008D. The optical depth in He I lines in the model of 24 January has been enhanced to mimic the effect of non-thermal excitations by fast electrons generated in the decay of ${ }^{56} \mathrm{Ni}$. While we cannot constrain the abundance of He this way this method allows us to identify He lines in the spectrum. The first two spectra are contaminated by the emission of the afterglow which is not described by the model. Therefore, the luminosity needed to match those spectra is too high to give a consistent description leading to an over-ionization of most species. This affects in particularly the Ca II IR triplet near $8000 \AA$, which is not reproduced by the models in the first two epochs. 


\section{References.}

1. van den Bergh, S., Li, W., \& Filippenko, A. V. PASP, 115, 1280 (2003).

2. Fruchter, A., et al. , Nature441, 463-468 (2006).

3. Veilleux, S., \& Osterbrock, D. E., ApJS, 63, 295 (1987).

4. Mannucci, F., et al. , A\&A, 401, 519 (2003).

5. Ulvestad, J. S., \& Ho, L. C. ApJ, 581, 925 (2002).

6. Clocchiatti, A., \& Wheeler, J.C. ApJ, 491, 375 (1997).

7. Page, K.L., et al. GCN 7164 (2008).

8. Schlegel, D.J., Finkbeiner, D.P., \& Davis, M., ApJ, 500, 525 (1998).

9. Immler, S., et al. GRB Coordinates Network, 7168, 1 (2008).

10. Li, W., Chornock, R., Foley, R. J., Filippenko, A. V., Modjaz, M., Poznanski, D., \& Bloom, J. S. GRB Coordinates Network, 7176, 1 (2008).

11. Li, L.-X., astro-ph 0803.0079 (2008).

12. Mazzali, P. A. \& Lucy, L. B., A\&A, 279, 447 (1993).

13. Lucy, L. B., A\&A, 345, 211 (1999).

14. Mazzali, P. A., A\&A, 363, 705 (2000).

15. Stehle, M., Mazzali, P. A., Benetti, S., \& Hillebrandt, W., MNRAS, 360, 1231 (2005).

16. Mazzali, P. A., et al., ApJ, 572, L61 (2002).

17. Mazzali, P. A., et al., Nature, 442, 1018 (2006).

18. Sauer, D. N., et al., MNRAS, 369, 1939 (2006).

19. Valenti, S., et al., ApJ, 673, L155 (2008). 